\title{
The role of the exocyst in renal ciliogenesis, cystogenesis, tubulogenesis, and development
}

\author{
Joshua H. Lipschutz ${ }^{1,2}$ (i) \\ 'Department of Medicine, Medical University of South Carolina, Charleston, SC, USA \\ ²Department of Medicine, Ralph H. Johnson Veterans Affairs Medical Center, Charleston, SC, USA
}

\begin{abstract}
The exocyst is a highly conserved eight-subunit protein complex (EXOC1-8) involved in the targeting and docking of exocytic vesicles translocating from the trans-Golgi network to various sites in renal cells. EXOC5 is a central exocyst component because it connects EXOC6, bound to the vesicles exiting the trans-Golgi network via the small GTPase RAB8, to the rest of the exocyst complex at the plasma membrane. In the kidney, the exocyst complex is involved in primary ciliognesis, cystogenesis, and tubulogenesis. The exocyst, and its regulators, have also been found in urinary extracellular vesicles, and may be centrally involved in urocrine signaling and repair following acute kidney injury. The exocyst is centrally involved in the development of other organs, including the eye, ear, and heart. The exocyst is regulated by many different small GTPases of the RHO, RAL, RAB, and ARF families. The small GTPases, and their guanine nucleotide exchange factors and GTPase-activating proteins, likely give the exocyst specificity of function. The recent development of a floxed Exoc5 mouse line will aid researchers in studying the role of the exocyst in multiple cells and organ types by allowing for tissue-specific knockout, in conjunction with Cre-driver mouse lines.
\end{abstract}

Keywords: Ciliogenesis, Exocyst, Exocytosis, Sec6/8 complex

\section{Introduction}

The highly-conserved exocyst complex comprises eight proteins, Exoc1-8 (originally named Sec3, Sec5, Sec6, Sec8, Sec10, Sec15, Exo70, and Exo84). The exocyst was first identified in the budding yeast, Saccharomyces cerevisiae. The six Sec proteins, named because mutations

Received April 30, 2019; Revised May 13, 2019;

Accepted May 20, 2019

Edited by Gheun-Ho Kim, Hanyang University, Seoul, Korea

Correspondence: Joshua H. Lipschutz

Renal Division, Department of Medicine, Medical University of South Carolina, 96 Jonathan Lucas Street, CSB 829, Charleston, SC 29425, USA. E-mail: Lipschut@musc.edu

Copyright (C) 2019 by The Korean Society of Nephrology

(a) This is an open-access article distributed under the terms of the Creative Commons Attribution Non-Commercial License (http://creativecommons. org/licenses/by-nc-nd/4.0/), which permits unrestricted non-commercial use, distribution, and reproduction in any medium, provided the original work is properly cited. inhibited secretion (or exocytosis), were discovered by Novick, Field, and Schekman almost four decades ago in their classic genetic screen. These investigators had a simple, yet elegant idea, that if the mother cell could not secrete into the daughter cell, then $S$. cerevisiae mutant cells that were temperature-sensitive for secretion and cell surface growth would become heavier during incubation at the non-permissive temperature $\left(37^{\circ} \mathrm{C}\right)$. This would allow for the selection of mutants by sedimentation of mutagenized cells on a Ludox density gradient [1]. This work was at least partially responsible for Dr. Randy Schekman being awarded the Nobel Prize in Physiology or Medicine in 2013. TerBush and Novick [2] later purified the exocyst protein complex containing Exoc1-6 (the six Sec proteins) and two additional subunits, Exoc7 and Exoc8 (Exo70 and Exo84, respectively). Mammalian homologues of all eight yeast exocyst proteins were identified from rat brains by Hsu et al [3] in 1996. All of the exocyst components are hydrophilic proteins which 
mutually interact to form a 19.5S complex peripherally associated with the plasma membrane [4]. The exocyst is thought to act as a holocomplex.

\section{Regulation of the exocyst complex}

In yeast, mutants of individual exocyst proteins accumulate vesicles in the cells because the vesicles cannot dock or fuse with the plasma membrane. The exocyst proteins localize to regions of active cell surface expansion, the bud tip at the beginning of the cell cycle and the mother-daughter cell connection during cytokinesis. Therefore, the exocyst complex is considered involved in directing vesicles to their precise sites of fusion [5-8].

The exocyst plays a central role in exocytosis; however, how the exocyst is regulated remains unclear. The exocyst, due to its complexity and ubiquity, potentially integrates many different inputs. Recent data from our laboratory and other studies have shown multiple small GTPases regulate the exocyst, including members of the Rab [6], Rho [9-14], Ral [15-18], and Arf [19,20] families (Table 1). Guanine nucleotide exchange factors (GEFs) of the small GTPases, such as Tuba, a GEF for Cdc42 [14,21], and Sec2 (mammalian homolog is Rabin8), a GEF for Sec4 (mammalian homolog is Rab8) [22], have also been shown to regulate exocyst (Table 1).

The first small GTPase found to interact with the exocyst complex was Sec4 (mammalian homolog Rab8), the founding member of the Rab family (Fig. 1) [6]. Rabs are important regulators of all vesicular trafficking events and Sec4 is essential for a post-Golgi event in yeast secretion. Genetic analysis indicated Sec4 functions upstream of the exocyst. The exocyst component Exoc6, specifically associates with secretory vesicles and interacts with Sec4-

Table 1. Small GTPases and GEFs shown to regulate the exocyst

\begin{tabular}{llc}
\hline \multicolumn{1}{c}{ GTPase } & \multicolumn{1}{c}{ Family } & Reference \\
\hline Rab8 (Sec4) & Rab & {$[6]$} \\
Rabin8 (Sec2) & GEF for Rab8 & {$[22]$} \\
Arf6 & Arf & {$[19]$} \\
Arl13 & Arf & {$[20]$} \\
RalA & Ral & {$[15-18]$} \\
Rho1 & Rho & {$[5]$} \\
Rho3 & Rho & {$[9,12]$} \\
Cdc42 & Rho & {$[13]$} \\
Tuba & GEF for Cdc42 & {$[14,21]$} \\
\hline
\end{tabular}

GEF, guanine nucleotide exchange factor.
GTP, which is found on the surface of the vesicular membrane. The interaction of Sec4-GTP with Exoc6 triggers further interactions between Exoc6 and other exocyst components, eventually leading to docking and fusion of vesicles with specific domains in the plasma membrane.

Because the exocyst localizes specifically to regions of active secretion and cell growth, how this localization is controlled is important. The second class of GTPases found to interact with the exocyst are members of the Rho family. Several rhol mutant alleles were identified in a search for mutants regulating the localization of green fluorescent protein (GFP)-tagged exocyst subunits in budding yeast [11]. The best-known Rho family function is organizing the actin cytoskeleton; however, Rhol exerts different effects on the exocyst via direct interaction between Rhol-GTP and the exocyst component Exoc1, which has been proposed as a "landmark" for defining polarized domains in the plasma membrane [5]. Another Rho family protein, Cdc42, was also shown to interact with Exocl and was required for the initial targeting of Exocl to the emerging yeast bud. Both Rhol and Cdc42 interact with the Exoc1 N-terminus and mutually compete for Exocl binding in vitro. Possibly, Cdc42 and Rhol

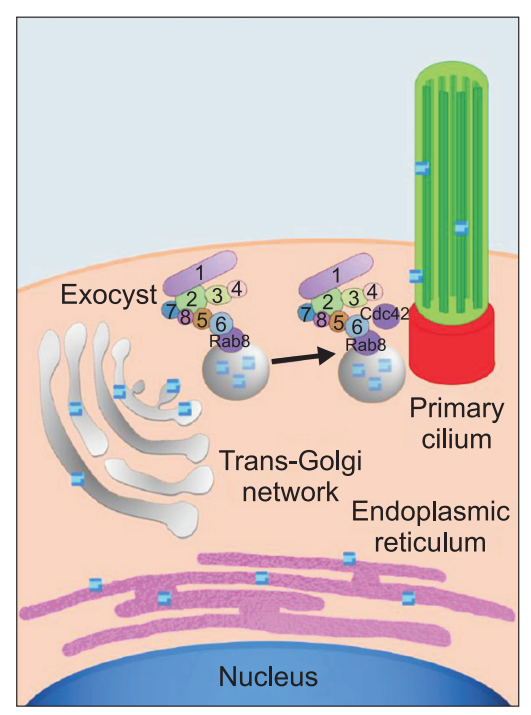

Figure 1. Model showing the involvement of the exocyst in ciliogenesis. Genes are transcribed into messenger RNA (mRNA) in the nucleus, and mRNA is translated into proteins in the endoplasmic reticulum. Proteins destined for the primary cilium are packaged in vesicles in the trans-Golgi network and trafficked to the primary cilium by the exocyst complex. The small GTPase Cdc42 localizes the exocyst to the primary cilium. Exoc5 is a central exocyst member because it connects Exoc6 (bound to the vesicle via Rab8) to the rest of the exocyst complex. Adapted from reference [36]. 
interact with Exocl at different stages of the yeast cell cycle in vivo [13].

In addition to the direct interaction of Rhol and Cdc42 with Exoc1, another Rho family protein, Rho3, was shown to associate with Exoc7, a different exocyst subunit $[9,12]$. The interaction between Rho3 and Exoc7 was greatly reduced when mutations were introduced into the Rho3 effector domain. The interaction between Rho3 and Exoc7 was dependent on the presence of Rho3-GTP [12]. Mutations in the Rho3 effector domain revealed roles in the regulation of actin organization, transport of exocytic vesicles to the bud, and docking of vesicles with the plasma membrane. Reportedly, Exoc7 mediates vesicle docking [9].

The mammalian exocyst (Sec6/8 complex) also localizes to areas of active exocytosis. In developing neurons, the exocyst localizes to growth cones and the tips of growing neurites [23]. In renal epithelial cells, the exocyst is concentrated near the tight junctions, a postulated region of active basolateral membrane addition [4], and the primary cilium [24], and regulates transport of vesicles between the trans-Golgi network (TGN) and plasma membrane [25]. When cultured in three-dimensional (3D) collagen gels, Madin-Darby canine kidney (MDCK) epithelial cells form multicellular cysts and, in response to hepatocyte growth factor, form tubules. This in-vitro system is a good model for studying the molecular pathways by which epithelial cells form these higher order structures $[26,27]$. Using this system, we showed the exocyst complex relocalized concomitant with changes in cell polarity that occur during these processes. Moreover, overexpression of the exocyst subunit Exoc5 specifically increased the synthesis of secretory and basolateral proteins, as well as the formation of cysts and tubules, indicating the exocyst is centrally involved in these higher order processes [28].

\section{The exocyst is centrally involved in ciliogenesis}

As stated above, the exocyst is involved in multiple cellular processes including basolateral transport [4,28], ciliogenesis [29], and protein translation in the endoplasmic reticulum $[30,31]$. Therefore, determining the mechanism by which the exocyst is involved in these processes is important. The involvement of the exocyst in primary ciliogenesis is particulary important because primary cil- ia are centrally involved in the pathogenesis of autosomal dominant polycystic kidney disease (ADPKD), the most common, potentially lethal genetic disease in humans, affecting 12,000,000 patients worldwide. In ADPKD, massive cystogenesis mechanically destroys the kidneys [32].

In 2017, the crystal structure of the central exocyst component, EXOC5, was solved [33], and an in-vivo 3D integrative approach to the exocyst was performed [34]. The details of the exocyst complex structure using cryoelecron microscopy was recently reported [35]. EXOC5 contains a VxPx ciliary targeting sequence that is highly conserved from yeast to humans. We analyzed solvent accessibility of the Val666, Ala667, and Pro668 residues of EXOC5 represented by the $5 \mathrm{~h} 11$ structure [33]. Solventaccessible surface areas were 37,49 , and $52 \AA^{2}$ and relative accessibilities were $32 \%, 73 \%$, and $50 \%$, respectively. Thus, all three residues are exposed to solvent and available for binding, although proline to a greater degree than valine. Therefore, we performed site-directed mutagenesis of EXOC5-myc cDNA in a pcDNA3 vector, mutating the cytosine at position 2002 to a guanine (cca to gca), causing translation of alanine instead of proline. Successful site-directed mutagenesis was confirmed by sequencing the full cDNA transcript. The pcDNA3 vector containing the mutated human EXOC5-myc ciliary targeting sequence was transfected into MDCK cells and stable cell lines generated. Three clonal cell lines expressing the mutated human EXOC5-myc ciliary targeting sequence were identified (G5, G7, and G9) using an antibody we generated against human EXOC5 [29].

The EXOC5 ciliary targeting sequence-mutated protein was confirmed stable and able to bind other members of the exocyst complex. Next, stably controlled, EXOC5overexpressing (OE), Exoc5 knockdown (KD), and EXOC5 ciliary targeting sequence-mutated MDCK cells, were cultured on Transwell filters. Results showed primary ciliogenesis was increased in EXOC5 OE cells and inhibited in Exoc5 KD and EXOC5 ciliary targeting sequencemutated cells. Next, the EXOC5 OE, Exoc5 KD, EXOC5 ciliary targeting sequence-mutated, and control MDCK cells, were grown in collagen gels until the cyst stage. Results showed EXOC5 OE cells formed mature cysts with single lumens more rapidly than control cysts, and Exoc5 KD and EXOC5 ciliary targeting sequence-mutated MDCK cells failed to form mature cysts, indicating the exocyst, acting through the primary cilium, is necessary 
for cystogenesis. Hepatocyte growth factor was added to induce tubulogenesis in the EXOC5 OE, Exoc5 KD, EXOC5 ciliary targeting sequence-mutated, and control cell cysts. EXOC5 OE cell cysts formed tubules more efficiently than control MDCK cell cysts. EXOC5 ciliary targeting sequence-mutated MDCK cell cysts formed significantly fewer tubules than control cell cysts, and Exoc5 $\mathrm{KD}$ cysts did not undergo tubulogenesis, indicating the exocyst, acting through the primary cilium, is necessary for renal tubulogenesis. Finally, EXOC5 messenger RNA (mRNA) completely rescued the ciliary phenotypes in exoc5 mutant zebrafish, and the EXOC5 ciliary targeting sequence-mutated mRNA could no longer efficiently rescue the phenotypes. Taken together, these data showed the exocyst, acting through the primary cilia, was necessary for renal ciliogenesis, cystogenesis, tubulogenesis, and development (Fig. 1) [36].

\section{Exoc5 floxed mice}

Because Exoc4 global knockout mice die very early during embryogenesis (during gastrulation) [37], a floxed exocyst mouse line was generated to study the function of the exocyst in murine kidneys. To the best of our knowledge, in 2015 we generated the first and only exocyst floxed mouse, Exoc $5^{\mathrm{fl} / \mathrm{fl}}$ [38]. Kidney-specific Exoc5 knockout mice that survived for 30 days had cystic kidney disease [39].

\section{The exocyst is involved in other renal processes and diseases}

We hypothesized that different small GTPases found at different locations in the cell, give the exocyst specificity of function. Using cell culture, zebrafish, and kidneyspecific knockout mice, Cdc42, a Rho family member, was found at the primary cilium and shown to regulate the exocyst [10]. Similarily, Tuba, a ciliary Cdc42 GEF, regulates the exocyst and is necessary for proper ciliogenesis, cystogenesis, and tubulogenesis [14,21]. Arl13b, an Arf family member, in its GTP form was shown to regulate the exocyst. Arl13b and Cdc42 genetically interact in zebrafish, and knockout of Arl13b in mice leads to renal cystogenesis, which phenocopies mice surviving for 30 days after kidney-specific knockout of Exoc5 [20]. Because multiple small GTPases appear to regulate the exocyst at the primary cilium, the exocyst, in addition to trafficking vesicles to the primary cilium, may have other function(s) in the primary cilium (e.g., secretion or retrieval of small extracellular vesicles). We have previously shown the exocyst, as well as regulators of the exocyst, are found in human urinary extracellular vesicles [40].

Another function of the exocyst in the kidney may be a role in the injury and/or recovery of renal tubule epithelial cells. Exoc5 overexpression in MDCK cells protects the cells from injury, which is mediated through the MAPK pathway [41-43]. This may involve primary cilia because unilateral nephrectomy in mice elongates primary cilia in the remaining kidney [44]. We are currently generating proximal-tubule specific Exoc5 OE and knockout mice to test this hypothesis. Preliminary results show that proximal tubule-specific Exoc5 knockout mice are significantly more susceptible to ischemia/reperfusion injury than control littermate mice (unpublished data).

In addition, we examined the role of the exocyst in podocyte development and disease. We identified two patients with exocyst deletions. In addition, all podocytespecific Exoc5 knockout mice died with 30 days and exhibited severe proteinuria and renal failure. This was a cilia-independent exocyst effect, as podocyte-specific intraflagellar protein 88 (Ift88) knockout mice did not have proteinuria or a phenotype [45].

\section{The exocyst in other organs}

Due to the ubiquitous expression of the exocyst complex, we hypothesized the exocyst affects ciliogenesis in other organs. After examining exoc5 knockout zebrafish [46], and generating photoreceptor-specific Exoc5 knockout mice (photoreceptors are modified primary cilia) [47], the exocyst, regulated by Cdc42, was shown to be necessary for eye development. Photoreceptor-specific knockout of Exoc5 in mice resulted in blindness [47]. Our collaborators in South Korea showed the exocyst also appeared necessary for cilia development in the ear [48]. Finally, patients with ADPKD have abnormal cardiac valves [49], especially biscuspid aortic valves (BAV) [50]. We recently showed that cardiac valves have cilia during development, but not in adulthood [51], and endocardial-specific Exoc5 knockout mice have BAV (unpublished data). 


\section{Summary}

The exocyst is a very important complex that targets and docks vesicles translocating from the TGN to various sites in renal cells, including the primary cilium. This complex appears to have many functions in the kidney that are likely a result of different small regulatory GTPases acting on the exocyst. By manipulating the exocyst and/or its regulators, many renal diseases and possibly diseases affecting other organs, could be treated.

\section{Conflicts of interest}

The author has no conflicts of interest to declare.

\section{Acknowledgments}

This work was supported in part by grants from the Veterans Affairs (Merit Award I01 BX000820), NIH (P30DK074038), and the American Heart Association (AWRP Winter 2017 Collaborative Sciences Award).

\section{References}

[1] Novick P, Field C, Schekman R. Identification of 23 complementation groups required for post-translational events in the yeast secretory pathway. Cell 1980;21:205-215.

[2] TerBush DR, Novick P. Sec6, Sec8, and Sec15 are components of a multisubunit complex which localizes to small bud tips in Saccharomyces cerevisiae. J Cell Biol 1995;130: 299-312.

[3] Hsu SC, Ting AE, Hazuka CD, et al. The mammalian brain rsec6/8 complex. Neuron 1996;17:1209-1219.

[4] Grindstaff KK, Yeaman C, Anandasabapathy N, et al. Sec6/8 complex is recruited to cell-cell contacts and specifies transport vesicle delivery to the basal-lateral membrane in epithelial cells. Cell 1998;93:731-740.

[5] Finger FP, Hughes TE, Novick P. Sec3p is a spatial landmark for polarized secretion in budding yeast. Cell 1998;92:559571.

[6] Guo W, Roth D, Walch-Solimena C, Novick P. The exocyst is an effector for Sec4p, targeting secretory vesicles to sites of exocytosis. ЕMBO J 1999;18:1071-1080.

[7] Lipschutz JH, Mostov KE. Exocytosis: the many masters of the exocyst. Curr Biol 2002;12:R212-R214.

[8] TerBush DR, Maurice T, Roth D, Novick P. The Exocyst is a multiprotein complex required for exocytosis in Saccharomyces cerevisiae. EMBO J 1996;15:6483-6494.

[9] Adamo JE, Rossi G, Brennwald P. The Rho GTPase Rho3 has a direct role in exocytosis that is distinct from its role in actin polarity. Mol Biol Cell 1999;10:4121-4133.

[10] Choi SY, Chacon-Heszele MF, Huang L, et al. Cdc42 deficiency causes ciliary abnormalities and cystic kidneys. $J$ Am Soc Nephrol 2013;24:1435-1450.

[11] Guo W, Tamanoi F, Novick P. Spatial regulation of the exocyst complex by Rhol GTPase. Nat Cell Biol 2001;3:353360.

[12] Robinson NG, Guo L, Imai J, Toh-E A, Matsui Y, Tamanoi F. Rho3 of Saccharomyces cerevisiae, which regulates the actin cytoskeleton and exocytosis, is a GTPase which interacts with Myo2 and Exo70. Mol Cell Biol 1999;19:3580-3587.

[13] Zhang X, Bi E, Novick P, et al. Cdc42 interacts with the exocyst and regulates polarized secretion. J Biol Chem 2001; 276:46745-46750.

[14] Zuo X, Fogelgren B, Lipschutz JH. The small GTPase Cdc42 is necessary for primary ciliogenesis in renal tubular epithelial cells. J Biol Chem 2011;286:22469-22477.

[15] Brymora A, Valova VA, Larsen MR, Roufogalis BD, Robinson PJ. The brain exocyst complex interacts with RalA in a GTP-dependent manner: identification of a novel mammalian Sec3 gene and a second Sec15 gene. J Biol Chem 2001; 276:29792-29797.

[16] Moskalenko S, Henry DO, Rosse C, Mirey G, Camonis JH, White MA. The exocyst is a Ral effector complex. Nat Cell Biol 2002;4:66-72.

[17] Polzin A, Shipitsin M, Goi T, Feig LA, Turner TJ. Ral-GTPase influences the regulation of the readily releasable pool of synaptic vesicles. Mol Cell Biol 2002;22:1714-1722.

[18] Sugihara K, Asano S, Tanaka K, Iwamatsu A, Okawa K, Ohta Y. The exocyst complex binds the small GTPase RalA to mediate filopodia formation. Nat Cell Biol 2002;4:73-78.

[19] Prigent M, Dubois T, Raposo G, et al. ARF6 controls postendocytic recycling through its downstream exocyst complex effector. J Cell Biol 2003;163:1111-1121.

[20] Seixas C, Choi SY, Polgar N, et al. Arl13b and the exocyst interact synergistically in ciliogenesis. Mol Biol Cell 2016;27: 308-320.

[21] Baek JI, Kwon SH, Zuo X, Choi SY, Kim SH, Lipschutz JH. Dynamin binding protein (Tuba) deficiency inhibits ciliogenesis and nephrogenesis in vitro and in vivo. $J$ Biol Chem 2016;291:8632-8643.

[22] Feng S, Knödler A, Ren J, et al. A Rab8 guanine nucleotide 
exchange factor-effector interaction network regulates primary ciliogenesis. J Biol Chem 2012;287:15602-15609.

[23] Hazuka CD, Foletti DL, Hsu SC, Kee Y, Hopf FW, Scheller $\mathrm{RH}$. The sec6/8 complex is located at neurite outgrowth and axonal synapse-assembly domains. J Neurosci 1999;19: 1324-1334.

[24] Rogers KK, Wilson PD, Snyder RW, et al. The exocyst localizes to the primary cilium in MDCK cells. Biochem Biophys Res Commun 2004;319:138-143.

[25] Yeaman C, Grindstaff KK, Wright JR, Nelson WJ. Sec6/8 complexes on trans-Golgi network and plasma membrane regulate late stages of exocytosis in mammalian cells. J Cell Biol 2001;155:593-604.

[26] O'Brien LE, Tang K, Kats ES, Schutz-Geschwender A, Lipschutz JH, Mostov KE. ERK and MMPs sequentially regulate distinct stages of epithelial tubule development. Dev Cell 2004;7:21-32.

[27] Pollack AL, Runyan RB, Mostov KE. Morphogenetic mechanisms of epithelial tubulogenesis: MDCK cell polarity is transiently rearranged without loss of cell-cell contact during scatter factor/hepatocyte growth factor-induced tubulogenesis. Dev Biol 1998;204:64-79.

[28] Lipschutz JH, Guo W, O'Brien LE, Nguyen YH, Novick P, Mostov KE. Exocyst is involved in cystogenesis and tubulogenesis and acts by modulating synthesis and delivery of basolateral plasma membrane and secretory proteins. Mol Biol Cell 2000;11:4259-4275.

[29] Zuo X, Guo W, Lipschutz JH. The exocyst protein Sec10 is necessary for primary ciliogenesis and cystogenesis in vitro. Mol Biol Cell 2009;20:2522-2529.

[30] Lipschutz JH, Lingappa VR, Mostov KE. The exocyst affects protein synthesis by acting on the translocation machinery of the endoplasmic reticulum. J Biol Chem 2003;278:2095420960.

[31] Toikkanen JH, Miller KJ, Söderlund H, Jäntti J, Keränen S. The beta subunit of the Sec61p endoplasmic reticulum translocon interacts with the exocyst complex in Saccharomyces cerevisiae. J Biol Chem 2003;278:20946-20953.

[32] Smyth BJ, Snyder RW, Balkovetz DF, Lipschutz JH. Recent advances in the cell biology of polycystic kidney disease. Int Rev Cytol 2003;231:51-89.

[33] Chen J, Yamagata A, Kubota K, Sato Y, Goto-Ito S, Fukai S. Crystal structure of Sec10, a subunit of the exocyst complex. Sci Rep 2017;7:40909.

[34] Picco A, Irastorza-Azcarate I, Specht T, et al. The in vivo architecture of the exocyst provides structural basis for exo- cytosis. Cell 2017;168:400-412.

[35] Mei K, Li Y, Wang S, et al. Cryo-EM structure of the exocyst complex. Nat Struct Mol Biol 2018;25:139-146.

[36] Zuo X, Lobo G, Fulmer D, et al. The exocyst acting through the primary cilium is necessary for renal ciliogenesis, cystogenesis, and tubulogenesis. J Biol Chem 2019;294:67106718.

[37] Friedrich GA, Hildebrand JD, Soriano P. The secretory protein Sec8 is required for paraxial mesoderm formation in the mouse. Dev Biol 1997;192:364-374.

[38] Fogelgren B, Polgar N, Lui VH, et al. Urothelial defects from targeted inactivation of exocyst sec10 in mice cause ureteropelvic junction obstructions. PLoS One 2015;10: e0129346.

[39] Polgar N, Lee AJ, Lui VH, Napoli JA, Fogelgren B. The exocyst gene Sec10 regulates renal epithelial monolayer homeostasis and apoptotic sensitivity. Am J Physiol Cell Physiol 2015;309:C190-C201.

[40] Chacon-Heszele MF, Choi SY, Zuo X, Baek JI, Ward C, Lipschutz JH. The exocyst and regulatory GTPases in urinary exosomes. Physiol Rep 2014;2:e12116.

[41] Fogelgren B, Zuo X, Buonato JM, et al. Exocyst Sec10 protects renal tubule cells from injury by EGFR/MAPK activation and effects on endocytosis. Am J Physiol Renal Physiol 2014;307:F1334-F1341.

[42] Jang HS, Han SJ, Kim JI, Lee S, Lipschutz JH, Park KM. Activation of ERK accelerates repair of renal tubular epithelial cells, whereas it inhibits progression of fibrosis following ischemia/reperfusion injury. Biochim Biophys Acta 2013; 1832:1998-2008.

[43] Park KM, Fogelgren B, Zuo X, Kim J, Chung DC, Lipschutz JH. Exocyst Sec10 protects epithelial barrier integrity and enhances recovery following oxidative stress, by activation of the MAPK pathway. Am J Physiol Renal Physiol 2010;298: F818-F826.

[44] Han SJ, Jang HS, Kim JI, Lipschutz JH, Park KM. Unilateral nephrectomy elongates primary cilia in the remaining kidney via reactive oxygen species. Sci Rep 2016;6:22281.

[45] Nihalani D, Solanki AK, Arif E, et al. Disruption of the exocyst induces podocyte loss and dysfunction. J Biol Chem 2019;294:10104-10119.

[46] Choi SY, Baek JI, Zuo X, Kim SH, Dunaief JL, Lipschutz JH. Cdc42 and sec10 are required for normal retinal development in zebrafish. Invest Ophthalmol Vis Sci 2015;56:33613370.

[47] Lobo GP, Fulmer D, Guo L, et al. The exocyst is required for 
photoreceptor ciliogenesis and retinal development. J Biol Chem 2017;292:14814-14826.

[48] Lee B, Baek JI, Min H, et al. Exocyst complex member EXOC5 is required for survival of hair cells and spiral ganglion neurons and maintenance of hearing. Mol Neurobiol 2018;55:6518-6532.

[49] Lumiaho A, Ikäheimo R, Miettinen R, et al. Mitral valve prolapse and mitral regurgitation are common in patients with polycystic kidney disease type 1 . Am J Kidney Dis 2001;38:1208-1216.

[50] Leier CV, Baker PB, Kilman JW, Wooley CF. Cardiovascular abnormalities associated with adult polycystic kidney disease. Ann Intern Med 1984;100:683-638.

[51] Toomer KA, Fulmer D, Guo L, et al. A role for primary cilia in aortic valve development and disease. Dev Dyn 2017; 246:625-634. 